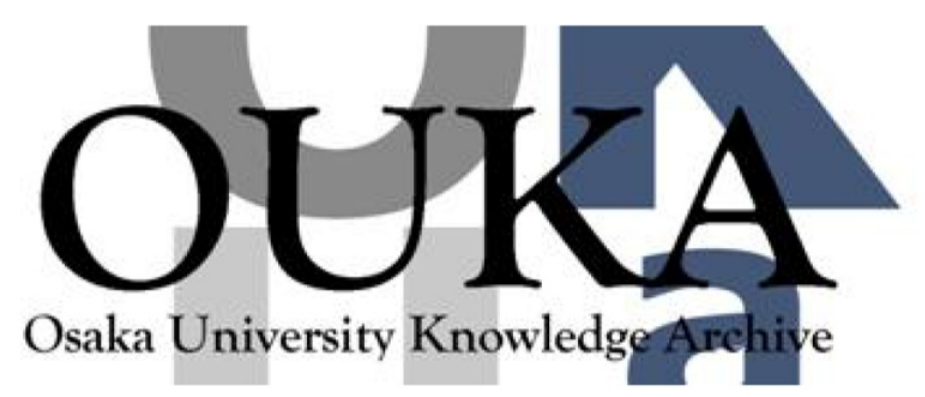

\begin{tabular}{|c|l|}
\hline Title & $\begin{array}{l}\text { Landau-Khalatnikov Damping of Ultrasound in } \\
\text { Heavy-Fermion Superconductors }\end{array}$ \\
\hline Author(s) & Miyake, K.; Varma, C. M. \\
\hline Citation & Physical Review Letters. 57(13) p. 1627-p. 1630 \\
\hline Issue Date & $1986-09-29$ \\
\hline oaire:version & VoR \\
\hline URL & $\begin{array}{l}\text { https://hdl. handle. net/11094/3417 } \\
\text { Miyake, K., Varma, C. M. Physical Review } \\
\text { Letters, } 57,13,1627-1630,1986-09-29 . \\
\text { "Copyright 1986 by the American Phys ical } \\
\text { Society." }\end{array}$ \\
\hline rights & \begin{tabular}{l} 
Note \\
\hline
\end{tabular} \\
\hline
\end{tabular}

Osaka University Knowledge Archive : OUKA

https://ir. Library. osaka-u. ac. jp/

0saka University 


\title{
Landau-Khalatnikov Eamping of Ultrasound in Heavy-Fermion Superconductors
}

\author{
K. Miyake ${ }^{(a)}$ and C. M. Varma \\ AT\&T Bell Laboratories, Murray Hill, New Jersey 07974
}

(Received 11 March 1986)

\begin{abstract}
The Landau-Khalatnikov mechanism associated with the relaxation of the order-parameter amplitude gives a peak in the longitudinal ultrasonic attenuation $\alpha$ near $T_{c}$, which quantitatively accounts for the observed peak in $\mathrm{UBe}_{13}$ and $\mathrm{UPt}_{3}$. At lower temperatures this mechanism gives a power-law attenuation.
\end{abstract}

PACS numbers: $74.30 . \mathrm{Gn}, 72.10 . \mathrm{Bg}, 74.20 . \mathrm{Fg}$

Recent experiments of longitudinal ultrasonic attenuation in $\mathrm{UBe}_{13}{ }^{1}$ and $\mathrm{UPt}_{3}{ }^{2}$ have shown that there exists a peak just below the transition temperature $T_{c}$. It was suggested in Ref. 1 that such a peak might provide evidence of a collective mode associated with internal degrees of freedom of anisotropic pairing as in superfluid ${ }^{3} \mathrm{He}$. Such a peak has not been observed in ordinary (singlet isotropic) superconductors. ${ }^{3}$

In this paper, we show the following: (i) A peak can be explained by the Landau-Khalatnikov (LK) mechanism associated with the relaxational mode of the order-parameter amplitude.,5 This mechanism works in principle for all types of pairing. (But as explained later for ordinary isotropic pairing, its magnitude is much smaller.) So the attenuation peak itself does not provide evidence for anisotropic pairing. (ii) Also at low temperatures $T<<T_{c}$, the LK mechanism gives a nonnegligible contribution ${ }^{6} \alpha_{r} \propto T^{n}$ to the sound attenuation; the exponent $n$ depends on the nature of the pairing and the mechanism of collision between quasiparticles and impurities. ${ }^{7}$

The essence of the LK mechanism is to take into account the existence of the macroscopic degrees of freedom which are associated with the order parameter $\eta$ which tends to relax to its local equilibrium $\eta_{\mathrm{le}}$ as determined by the other hydrodynamical variables (in the present case, the dilatation $\delta$ of the lattice). Entropy production associated with the relaxation process $\eta \rightarrow \eta_{\mathrm{le}}(\delta)$ causes sound attenuation in general. The equation of motion for the dilatation $\delta$ is written in terms of the free-energy density $F(\delta, \eta)$ as

$$
\rho \frac{\partial^{2} \delta}{\partial t^{2}}=\frac{\partial^{2}}{\partial z^{2}} \frac{\partial F(\delta, \eta)}{\partial \delta},
$$

where $\rho$ is the mass density of the lattice and it is assumed that the longitudinal sound is propagating along $c(\| \hat{\mathbf{z}})$ axis. If $\eta_{\mathrm{le}}(\delta)$ were substituted into (1), it would reduce to the usual wave equation. The fluctuating part of the "pressure" $P \equiv \partial F(\delta, \eta) / \partial \delta$ consists of two parts:

$$
P^{\prime}=\left(\frac{\partial P}{\partial \delta}\right)_{\eta} \delta^{\prime}+\left(\frac{\partial P}{\partial \eta}\right)_{\delta} \eta^{\prime} .
$$

Hereafter primed quantities are assumed to vary as $\exp [i(k z-\omega t)]$. By assuming that the dilatation $\delta^{\prime}$ gives the change in the chemical potential $\mu^{\prime}$ of the electronic system, $\mu^{\prime} \propto \delta^{\prime}$, and that $k \xi_{0}<<1\left(\xi_{0}\right.$ being the coherence length) so that motion of $\eta$ is determined locally, it follows from linear response theory ${ }^{8,9}$ that

$$
\eta^{\prime k}(\omega)=\frac{\chi_{\eta n}(k, \omega)}{\chi_{\eta n}(k, 0)} \frac{d \eta}{d \delta} \delta^{\prime k}(\omega) .
$$

Here $\chi_{A B}(k, \omega)$ denotes a linear response function $\left\langle\left\langle A_{k}, B_{-k}\right\rangle\right\rangle(\omega)$ and $d \eta / d \delta$ is the isothermal derivative. Then, substituting (3) and (2) into (1), we obtain the velocity $s(\omega)$ and the attenuation $\alpha_{r}(\omega)$ :

$$
\begin{aligned}
& s(\omega) \simeq s_{0}-\frac{s_{\infty}^{2}-s_{0}^{2}}{2 s_{0}} \operatorname{Re} I(\omega), \\
& \alpha_{r}(\omega) \simeq \frac{s_{\infty}^{2}-s_{0}^{2}}{2 s_{0}^{3}} \operatorname{Im} I(\omega) \omega,
\end{aligned}
$$

where

$$
\begin{aligned}
& I(\omega) \equiv \lim _{k \rightarrow 0} \frac{\chi_{\eta n}(k, \omega)-\chi_{\eta n}(k, 0)}{\chi_{\eta n}(k, 0)}, \\
& s_{\infty}^{2} \equiv \frac{(\partial P / \partial \delta)_{\eta}}{\rho}
\end{aligned}
$$

and

$$
s_{\infty}^{2}-s_{0}^{2} \equiv \frac{1}{\rho}\left(\frac{\partial P}{\partial \eta}\right)_{\delta} \frac{d \eta}{d \delta} .
$$

In order to calculate $I(\omega)$, we assume a "polarlike" gap as a simple representative of those with nodes along lines on the Fermi surface, since the state with such a gap seems to explain the existing experiments consistently. ${ }^{7}$ We shall mention the results for the "axial-like" gap also.

Critical regime $\left[0<t \equiv 1-T / T_{c}<<1\right]$. -The response function $\chi_{\eta n}(k, \omega)$ with

$$
\eta_{k} \equiv \sum_{p} \hat{p}_{z}\left(a_{p+k / 2} a_{-p+k / 2}+\text { H.c. }\right) / \sqrt{2}
$$

and $n_{k} \equiv \sum_{p} a_{p-k / 2}^{\dagger} a_{p+k / 2}$ can be calculated easily with 
the random-phase approximation as in Refs. 8 and 9. In the critical regime, the main contribution to the fully renormalized collision rate $1 / \tau$ of quasiparticles is expected to come, for anisotropic pairing, from nonmagnetic impurity scattering and is approximated by $1 / \tau_{N}$ ( $\tau_{N}$ being the lifetime in the normal state). In the hydrodynamical region $\omega \tau<<1, \chi_{\eta n}(k, \omega)$ can be calculated analytically. ${ }^{8}$ The result for $I(\omega)$ is

$$
I(\omega)=i \omega \tau_{r}(T) /\left[1-i \omega \tau_{r}(T)\right]
$$

where the relaxation time $\tau_{r}(T)$ shows the so-called critical slowing down':

$$
\tau_{r}(T) \simeq \begin{cases}\frac{1}{16} \pi^{2}[10 / 7 \zeta(3)]^{1 / 2}, & \Delta(T) \tau_{N}>>1, \\ \frac{1}{48} \pi\left(\hbar / k_{\mathrm{B}} T_{c}\right) t^{-1}, & \Delta(T) \tau_{N}<<1 .\end{cases}
$$

Equation (7) has the familiar Debye form but with a lifetime determined by the critical fluctuations. The maximum gap $\Delta(T)$ is defined by $\Delta_{p} \equiv \Delta \hat{p}_{z}$. With use of (7), the sound velocity (4) and attenuation (5) are written as

$$
\begin{aligned}
& s(\omega) \simeq s_{0}+\frac{s_{\infty}^{2}-s_{0}^{2}}{2 s_{0}} \frac{\omega^{2} \tau_{r}^{2}}{1+\omega^{2} \tau_{r}^{2}}, \\
& \alpha_{r}(\omega) \simeq \frac{s_{\infty}^{2}-s_{0}^{2}}{2 s_{0}^{3}} \frac{\omega^{2} \tau_{r}}{1+\omega^{2} \tau_{r}^{2}} .
\end{aligned}
$$

One can see from (9) and (10) that $\left(s_{\infty}^{2}-s_{0}^{2}\right) / 2 s_{0}$ is equal to the velocity change $\Delta s$ across the transition point, and that the attenuation $\alpha_{r}$ exhibits a peak around $\omega \tau_{r}(T)=1$ as a function of $T$ with fixed frequency $\omega$. The peak position is given by $t_{p} \equiv 1-T_{p} / T_{c}-\left(\omega \tau_{N}\right)^{2}$ for $\Delta\left(T_{p}\right) \tau_{N} \gg>1$ and $t_{p} \simeq\left(\pi \hbar \omega / 48 k_{\mathrm{B}} T_{c}\right)$ for $\Delta\left(T_{p}\right) \tau_{N}<<1$, respectively, which in turn makes it possible to determine $\tau_{N}$ from the peak position. This value can be checked against an independent measurement, say from the resistivity or the attenuation in the normal state. It should be remarked that the peak height $\alpha_{p}(\omega) \simeq\left(\Delta s / 2 s^{2}\right)_{T_{c}} \omega$ is determined completely by the sound velocity and its jump at $T_{c}$ and is proportional to $\omega$.

If we use the measured values of $\Delta s / s \sim 25 \times 10^{-6}$ $\left(13 \times 10^{-6}\right)$ and $s \sim 8 \times 10^{5} \mathrm{~cm} / \mathrm{sec}\left(3.9 \times 10^{5} \mathrm{~cm} / \mathrm{sec}\right)$ for $\mathrm{UBe}_{13}{ }^{1}\left(\mathrm{UPt}_{3}\right),{ }^{10}$ the peak height is predicted as $\alpha_{p}(\omega)=9.8 \times 10^{-2} \omega / 2 \pi \mathrm{cm}^{-1} \mathrm{GHz}\left(1.0 \times 10^{-1} \omega / 2 \pi\right.$ $\mathrm{cm}^{-1} \mathrm{GHz}$ ). When we compare these predictions with experiments, we have to take into account carefully the following two factors: (i) These compounds have rather wide transition widths $\delta T_{c}\left(-40 \mathrm{mK}\right.$ in $\mathrm{UBe}_{13}{ }^{1}$ and $-15 \mathrm{mK}$ in $\mathrm{UPt}_{\boldsymbol{e}}{ }^{2}$ ). Therefore, if $t_{p}<\delta T_{c} / T_{c}$, the peak is reduced considerably as a result of smearing. (ii) The observed attenuation $\alpha$ should be $\alpha=\alpha_{\mathrm{qp}}+\alpha_{r}$, where $\alpha_{\mathrm{qp}}$ is the contribution from quasiparticle collisions ${ }^{7,11}$ which decreases rather rapidly when going away from $T_{c}$ (say around $t \sim 10^{-1}$ ).
This contribution shifts the observed peak position closer to $T_{c}$ than that given by $t_{p}{ }^{12}$ With consideration of these two factors, the quantitative agreement between the above predictions for $\alpha_{p}(\omega)$ and the experiments in $\mathrm{UBe}_{13}{ }^{1}$ and $\mathrm{UPt}_{3}{ }^{2}$ is not bad (see Table I.) Discrepancies between the theory and the experiment in $\mathrm{UPt}_{3}$ for higher frequencies are due to the factor (ii), while those in $\mathrm{UBe}_{13}$ for lower frequencies are due to the factor (i). The values of $T_{c}-T_{p}$ used to get the theoretical numbers in Table $I$ are the observed values, $\approx 30 \mathrm{mK}$ at $0.092 \mathrm{GHz}$ in $\mathrm{UPt}_{3}$ and $50 \mathrm{mK}$ at $2.4 \mathrm{GHz}$ in $\mathrm{UBe}_{13}$. From these values and the relation (8), the lifetime $\tau_{N}$ is estimated as $\tau_{N} \sim 6 \times 10^{-10} \mathrm{sec}$ for $\mathrm{UPt}_{3}{ }^{2}$ and $\tau_{N}<5 \times 10^{-12} \mathrm{sec}$ for $\mathrm{UBe}_{13}, 1,13$ where $\tau_{N} \Delta\left(T_{p}\right) \sim 8$ for $\mathrm{UPt}_{3}$ and $\tau_{N} \Delta\left(T_{p}\right)<1$ for $\mathrm{UBe}_{13} \cdot{ }^{14}$ The position of the peak and its detailed shape depends on the value of $\Delta(T) \tau_{N}$. But the peak of $I(\omega)$ given in Table I occurs at $\omega \tau_{r}=1$. It is calculated from the observed change in sound velocity and does not depend on the value of $\Delta(T) \tau_{N}$.

In the regime near $T_{c}$, we can see the GinzburgLandau form for the free-energy density

$$
\begin{gathered}
F(\delta, \eta)=F_{0}(\delta)+a^{\prime}(\delta)\left(T-T_{c}\right) \eta^{2} \\
+b(\delta) \eta^{4}+\ldots
\end{gathered}
$$

Then, we can show that the quantity $s_{\infty}^{2}-s_{0}^{2}$, Eq. (6) is expressed in terms of thermodynamic quantities as

$$
s_{\infty}^{2}-s_{0}^{2}=s^{2} \frac{\Delta C_{p}}{T_{c}} B\left(\frac{d T_{c}}{d P}\right)^{2} \quad\left(T \simeq T_{c}\right),
$$

where $\Delta C_{p}, B$, and $P$ denote the specific-heat jump at $T_{c}$, the bulk modulus, and the pressure, respectively. We have verified that relation (11), with $\Delta s \simeq\left(s_{\infty}^{2}-s_{0}^{2}\right) / 2 s$, holds fairly well both in $\mathrm{UPt}_{3}{ }^{11}$ and $\mathrm{UBe}_{13}$.

The reason why the attenuation peak has not been observed in ordinary superconductors is as follows: In the case of isotropic pairing, the lifetime $\tau_{N}$ relevant to

TABLE I. Calculated and experimental magnitudes of the ultrasonic attenuation peak in $\mathrm{UPt}_{3}$ and $\mathrm{UBe}_{13}$.

\begin{tabular}{ccc}
\hline \hline $\begin{array}{c}f \\
(\mathrm{GHz})\end{array}$ & $\begin{array}{c}\text { Theory } \\
\alpha_{p}(\omega)\left(\mathrm{cm}^{-1}\right)\end{array}$ & $\begin{array}{c}\text { Experiment } \\
\alpha_{p}-\alpha_{N}\left(\mathrm{~cm}^{-1}\right)\end{array}$ \\
\hline \multicolumn{3}{c}{$\mathrm{UPt}_{3}{ }^{\mathrm{a}}$} \\
0.92 & 0.009 & 0.008 \\
0.15 & 0.015 & 0.102 \\
0.27 & 0.027 & 0.019 \\
& & \\
1.3 & 0.13 & 0.075 \\
1.7 & $\mathrm{UBe}_{13}{ }^{\mathrm{b}}$ & 0.014 \\
2.4 & 0.17 & 0.24 \\
\hline \hline
\end{tabular}

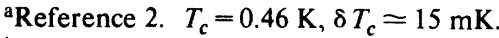

${ }^{\mathrm{b}}$ References 1 and 13. $T_{c}=0.86 \mathrm{~K}, \delta T_{c}=40 \mathrm{mK}$. 
the relaxation of the order parameter is not given by nonmagnetic impurity scattering (dynamical version of the Anderson theorem) but by the electron-phonon scattering, and is the order ${ }^{9}$ of $10^{-8} \mathrm{sec}$, much larger than the $\sim 10^{-10}-10^{-12} \mathrm{sec}$ for heavy-fermion systems. Then, in order that the peak position, determined in that case by $t_{p} \sim\left(\omega \tau_{N}\right)^{2}$, is located near $T_{c}$, the frequency $\omega$ should be much less than $2 \pi \times 10^{-8}$ $\sec ^{-1}$; so that the peak height $\alpha_{p}(\omega)=\left(\Delta s / 2 s^{2}\right) \omega$ becomes about $10^{-2}$ times smaller than those observed in $\mathrm{UPt}_{3}$ and $\mathrm{UBe}_{13}$, even if $\Delta s / s$ were of the same order of magnitude as in $\mathrm{UPt}_{3}$ and $\mathrm{UBe}_{13}$.

The relaxational mode of the order-parameter amplitude can also couple with the transverse sound in principle. However, its coupling is proportional to $(d \eta / d \delta)\left(d \delta / d \delta_{t}\right), \delta_{t}$ being the transverse deformation of the lattice [cf. Eq. (3)], so that the coupling is smaller by a factor $\left|\left(d \delta / d \delta_{t}\right)\right|<<1$ compared with the longitudinal sound. This is consistent with the fact that no attenuation peak near $T_{c}$ has been observed for the transverse sound. ${ }^{2}$

Since the compression of the lattice in a given direction causes a change in the anisotropy of the interaction and in the density of states of quasiparticles, the longitudinal sound can couple, in principle, with the "orbital" mode associated with the internal degrees of freedom of anisotropic Cooper pairs. This might lead one to think that the attenuation peak might be explained by resonance absorption with the "orbital" modes, whose eigenfrequency is $O(\Delta)$, as in superfluid ${ }^{3} \mathrm{He} .{ }^{15}$ In the hydrodynamic region, however, these "orbital" modes become purely damped, so that the attenuation is expected to be given by a formula similar to (5) and (7) with an appropriate relaxation time of the "orbital" mode. If crystal-field anisotropies are included, the mathematical structure is quite similar to that of the orbital dynamics in ${ }^{3} \mathrm{He}-A$, in which case the origin of the anisotropy was the dipole interaction between nuclear spins of ${ }^{3} \mathrm{He} .{ }^{8,16}$ Its relaxation time $\tau_{\text {orb }}$ can be estimated in a similar way to that above: The longest $\tau$ corresponds to the fluctuations of the "orbital" axis, i.e., $\Sigma_{p} \hat{p}_{x}\left(a_{p} a_{-p}+\right.$ H.c. $)$ and $(x \rightarrow y)$, and is given as

$$
\tau_{\text {orb }} \simeq \frac{\pi}{32} \frac{1-g_{a}}{g_{a} T_{c}} N(0) V_{0}\left\{\begin{array}{l}
\Delta(T) \tau_{N}, \quad \Delta(T) \tau_{N} \gg>1, \\
1, \quad \Delta(T) \tau_{N}<<1,
\end{array}\right.
$$

where $\left(1-g_{a}\right) V_{0}$, with $0<g_{a}<1$, is the attractive interaction for the wrong components $\hat{p}_{x}$ and $\hat{p}_{y}$ if the polarlike state $\Delta_{p}=\Delta \hat{p}_{z}$ is assumed. The relaxation time $\tau_{\text {orb }}$ shows critical speeding up in contrast with $\tau_{r}$, Eq. (8). The reason is that the fluctuations of the "orbital" axis couple with the external variable, in this case the anisotropy of the thermal distribution of quasiparticles; this gives an "orbital" viscosity of Cross-
Anderson type ${ }^{16}$ which vanishes more rapidly $\left(\sim \Delta^{3}\right)$ than the restoring force $\left(\sim \Delta^{2}\right)$ of the "orbital" axis when approaching $T_{c}$. Therefore, the resonance $\omega \tau_{\text {orb }}=1$ never occurs as long as $\omega \tau_{N}<<1$, i.e., in the hydrodynamical region. The Carlson-Goldman modes, ${ }^{17}$ which is essentially the phase mode of the order parameter, can also couple with the longitudinal sound in principle. However, the coupling is not large enough to give the observed peak: Indeed, the ratio between the peak value $\alpha_{\mathrm{CG}}$ due to the phase mode in the pure limit and $\alpha_{p}$ due to the amplitude mode is estimated as

$$
\begin{aligned}
\alpha_{\mathrm{CG}} / \alpha_{p}=[ & \left.56 \zeta(3) / 135 \pi^{3}\right]\left[N(0) V_{0}\right]^{2} \\
& \times\left(T_{c} \tau_{N}\right)^{2} \tau_{N} \omega\left[\Delta(T) / T_{q}\right]<<1
\end{aligned}
$$

for $\mathrm{UPt}_{3}{ }^{1}$ and $\mathrm{UBe}_{13} .{ }^{13}$

Low-temperature region $[T<\Delta(T)]$. - In the case $\omega \tau<<1$ and $T \tau>>1(\tau$ being the lifetime of quasiparticles in the superconducting state), $I(\omega)$ can be calculated similarly as for the critical regime. Recent analysis ${ }^{7}$ of the existing experiments shows that $\tau$ should be nearly temperature independent and of the order of $\tau_{N}$, which is in marked contrast with a naive expectation $\tau(E) \propto 1 / N(E), N(E)$ being the density of states of excitations. ${ }^{18,19}$ Here we assume according to Ref. 7 that $\tau$ is temperature independent. Then, $I(\omega)$ is calculated to be

$$
I(\omega)=i \omega \frac{81 \pi \zeta(3)}{16} \tau\left(\frac{T}{\Delta}\right)^{3}+\ldots .
$$

Substituting (13) into (5), we obtain ${ }^{20}$

$$
\alpha_{r}(\omega) \simeq g(T) \frac{\Delta s}{s^{2}\left(T_{c}\right)} \frac{81 \pi \zeta(3)}{16} \tau\left(\frac{T}{\Delta}\right)^{3} \omega^{2},
$$

where $g(T)$ is a slowly varying function with $g\left(T_{c}\right)=1$. With use of the experimental values for $\Delta s / s\left(T_{c}\right), \alpha_{N}\left(\alpha_{N}\right.$ being the attenuation at $\left.T_{c}\right)$, and the lifetime $\tau\left(\sim \tau_{N}\right.$ is estimated from the peak position of attenuation) the ratio $\alpha_{r} / \alpha_{N}$ is estimated as $\alpha_{r} / \alpha_{N} \sim 26 g(T / \Delta)^{3}$ and $6 g(T / \Delta)^{3}$ for $\mathrm{UBe}_{13}{ }^{1}$ and $\mathrm{UPt}_{3},{ }^{2}$ respectively. The $T^{3}$ dependence of $\alpha$ observed at $T$ down to $\sim T_{c} / 2$ in $\mathrm{UPt}_{3}{ }^{2}$ is in agreement with the above prediction, Eq. (14), although it should be remembered that other mechanisms ${ }^{7}$ also contribute in this temperature region.

If the gap is "axial-like," e.g., $\Delta_{p}=\Delta\left(\hat{p}_{x}+i \hat{p}_{y}\right)$, sound attenuation due to the LK mechanism at low temperatures is given in the form ${ }^{20}$

$$
\alpha_{r}(\omega) \simeq g(T) \frac{\Delta s}{s^{2}\left(T_{c}\right)} \frac{7 \pi^{4}}{75} \tau\left(\frac{T}{\Delta}\right)^{4} \omega^{2} .
$$

As far as the peak near $T_{c}$ is concerned, there is essentially no difference between the predictions for the "axial-like" and the "polarlike" states. 
We wish to acknowledge very useful and stimulating conversations with B. Batlogg, D. J. Bishop, B. Golding, and S. Schmitt-Rink.

(a) Permanent address: Department of Physics, Nagoya University, Chikusa-Ku, Nagoya 464, Japan.

${ }^{1}$ B. Golding, D. J. Bishop, B. Batlogg, W. H. Haemmerle, Z. Fisk, J. L. Smith, and H. R. Ott, Phys. Rev. Lett. 55, 2479 (1985).

${ }^{2}$ V. Müller, D. Maurer, E. Scheidt, Ch. Roth, K. Lüder, E. Bucher, and H. E. Bömmel, to be published.

${ }^{3}$ However, such a peak has been observed in $A 15$ compounds just below the superconducting transition point; see, e.g., T. Fukase, M. Tachiki, N. Toyota, and Y. Muto, Solid State Commun. 18, 505 (1976).

${ }^{4}$ L. D. Landau and I. M. Khalatnikov, Dokl. Akad. Nauk SSSR 96, 469 (1954) [English translation: in Collected papers of L. D. Landau, edited by D. ter Haar (Pergamon, London, 1965), p. 626].

5Ultrasonic attenuation due to the LK mechanism has been clearly observed in ${ }^{4} \mathrm{He}$ near the $\lambda$ point [see, e.g., R. D. Williams and I. Rudnick, Phys. Rev. Lett. 25, 276 (1970)] and the order-parameter-amplitude mode in the collisionless regime has been observed in $\mathrm{NbSe}_{2}$ [see, e.g., P. B. Littlewood and C. M. Varma, Phys. Rev. B 26, 4883 (1982)].

${ }^{6} \mathrm{~B}$. Batlogg (private communication) suggested to us that the low-temperature tail of the LK peak may be relevant to the experiments.

${ }^{7}$ At low temperatures other mechanisms contribute to attenuation. See S. Schmitt-Rink, K. Miyake, and C. M. Varma, to be published.

${ }^{8}$ Calculations can be performed in the way quite similar to H. Takagi and K. Miyake, Prog. Theor. Phys. 63, 772 (1980).

${ }^{9}$ The temperature dependence of $\tau_{r}(T)$, Eq. (8), is the same as that for single BCS pairing [see, e.g., E. Abrahams and T. Tsuneto, Phys. Rev. 152, 416 (1966); A. Schmidt, Phys. Kondens. Mater. 8, 129 (1968)] and also for "axiallike" pairing.

10B. Batlogg, D. J. Bishop, E. Bucher, and C. M. Varma, J. Appl. Phys. 57, 3060 (1985).

11J. P. Rodriguez, Phys. Rev. Lett. 55, 250 (1985).
12Indeed, the first measurement in $\mathrm{UPt}_{3}$ [D. J. Bishop, C. M. Varma, B. Batlogg, E. Bucher, Z. Fisk, and J. L. Smith, Phys. Rev. Lett. 53, 1009 (1984), in which a clear peak was not observed in $\alpha$ ] also shows existence of $\alpha$, if a proper subtraction $\alpha-\alpha_{\mathrm{qp}}$ is performed; see e.g., Fig. 1 of Ref. 11.

${ }^{13} \mathrm{~B}$. Golding, private communication.

${ }^{14}$ Considering the uncertainty about the Fermi velocity and the area of the Fermi surface, the values of $\tau_{N}$ thus estimated are consistent with those estimated for the attenuation just above $T_{c}$ : B. Batlogg, D. J. Bishop, B. Golding, E. Bucher, J. Hufnagl, Z. Fisk, J. L. Smith, and H. R. Ott, Phys. Rev. B 33, 5906 (1986). They are also consistent with resistivity measurements in the normal state.

${ }^{15}$ See, e.g., P. Wölfe, in Progress in Low Temperature Physics, edited by D. F. Brewer (North-Holland, Amsterdam, 1978), Vol. 7A, p. 191; D. S. Hirashima and H. Namaizawa, Prog. Theor. Phys. 74, 400 (1985).

${ }^{16}$ A. J. Leggett and S. Takagi, Ann. Phys. (N.Y.) 110, 353 (1978); M. C. Cross and P. W. Anderson, in Proceedings of the Fourteenth International Conference on Low-Temperature Physics, edited by M. Krusius and M. Vuorio (NorthHolland, Amsterdam, 1975), Vol. 1, p. 29.

${ }^{17}$ R. V. Carlson and A. M. Goldman, Phys. Rev. Lett. 34, 11 (1975). Goldman (private communication) has suggested that the observed peak in the heavy-fermion superconductors could be due to such a mode.

${ }^{18}$ L. Coffey, T. M. Rice, and K. Ueda, J. Phys. C 18, L813 (1985).

${ }^{19}$ However, the effect of multiple scattering with impurities could give an almost temperature-independent $\tau$ if the scattering phase shift $\delta_{F} \sim \pi / 2$ (see C. J. Pethick and D. Pines, to be published; S. Schmitt-Rink, private communication).

20If we used $\tau=\tau_{N} N_{F} / N(E)$ as the lifetime of quasiparticles, we would obtain

$$
I(\omega) \simeq i \omega\left(3 \pi^{2} / 8\right) \tau_{N}(T / \Delta)^{2}+\ldots
$$

for (13), and

$$
\alpha_{r}(\omega) \simeq g(T)\left[\Delta s / s^{2}\left(T_{c}\right)\right]\left(3 \pi^{2} / 8\right) \tau_{N}(T / \Delta)^{2} \omega^{2}
$$

for (14); in the case of "axial-like" gap,

$$
\alpha_{r}(\omega) \simeq g(T)\left[\Delta s / s^{2}\left(T_{c}\right)\right]\left(\pi^{2} / 15\right) \tau_{N}(T / \Delta)^{2} \omega^{2}
$$

for (15). 